\title{
Distally Based Pedicled Fibula Flap for Reconstruction of Infected Charcot's Midtarsal Collapse-Diabetic Rocker Bottom Foot
}

\author{
T. M. Balakrishnan ${ }^{1, \odot}$ Sathya Pakkiri ${ }^{1} \quad$ Arounkumar Nagalingam ${ }^{1} \quad$ R. Selvaraj ${ }^{2} \quad J$. Jaganmohan $^{1}$ \\ ${ }^{1}$ Department of Plastic and Faciomaxillary Surgery, Madras Medical \\ College, Chennai, Tamil Nadu, India \\ 2Department of Orthopedic Surgery, Institute of Orthopedic and \\ Traumatology, Madras Medical College, Chennai, Tamil Nadu, India \\ Address for correspondence T.M. Balakrishnan, MBBS, MS, \\ FRCS (E), DNB, MCH, DNB, Old no 15, New no 10, Thiruvalluvar \\ Street, Rangarajapuram, Kodambakkam, Chennai 600024, Tamil \\ Nadu, India (e-mail: thalaiviri.b@gmail.com).
}

Indian J Plast Surg:2021;54:20-28.

\author{
Abstract \\ Keywords \\ - charcot's joint \\ - diabetic foot \\ syndrome \\ - distally based pedicled \\ fibula flap \\ - infected diabetic \\ rocker bottom foot \\ - orthoplastic approach
}

\section{Introduction}

Charcot neuroarthropathy (CNAP) was first described in $1868 .{ }^{1}$ There is a pari passu increase in the diabetic CNAP deformities and CNAP-related amputations in the last 30 years with expanding diabetic world population. ${ }^{2-13}$ Diabetes mellitus has become the most common etiology for CNAP in the modern era. The reverberating downhill cycle of diabetic CNAP starts with the deformities leading to foot ulcers that precipitate infection and finally the amputation. ${ }^{14-16}$ The prevalence of diabetic CNAP is variably reported in literature ranging from 0.08 to $13 \% .{ }^{17}$ Higher occurrence of diabetic CNAP and severe peripheral neuropathy in those visiting diabetic foot care clinics are reported..$^{18}$ The author also observes a progressive increase in the incidence of diabetic CNAP for the past 20 years in his practice. In the year 2016, an epidemiological study was conducted
DOI https://doi.org/

10.1055/s-0040-1719197

ISSN 0970-0358. in the author's institute, in which $25.5 \%$ of patients attending the diabetic foot care unit were found to be affected by diabetic CNAP. Ten to fifteen percent of the patients with diabetes mellitus undergo lower extremity amputation in their lifetime for unsalvageable CNAP deformities. ${ }^{12} \mathrm{CNAP}$ is a lucid risk factor for a lower extremity amputation. The most common form of CNAP in the diabetic foot is the involvement of tarsometatarsal joint and naviculocuneiform joint (Broadsky type 1$).{ }^{19}$ This results in a fixed or mobile rocker bottom deformity with or without valgus angulation forefoot deformity. The collapse of the medial longitudinal and the transverse arches of the foot predispose to the development of hyperpression area in the medial border of the foot with faulty biomechanical recruitment of peroneal evertors. The forefoot goes into pronation that predisposes to the development of ulcers over the collapsed naviculocuneiform bony complex. The secondary infection of these (c) 2021. Association of Plastic Surgeons of India.

This is an open access article published by Thieme under the terms of the Creative Commons Attribution-NonDerivative-NonCommercial-License, permitting copying and reproduction so long as the original work is given appropriate credit. Contents may not be used for commercial purposes, or adapted, remixed, transformed or built upon. (https://creativecommons.org/licenses/by-nc-nd/4.0/).

Thieme Medical and Scientific Publishers Pvt. Ltd. A-12, 2nd Floor, Sector 2, Noida-201301 UP, India 
ulcers plays havoc in causing sequestration of the tarsal bony rows to a variable extent rendering pedal instability and even may convert the whole tarsum into a bag of bone and pus in our observation. This unpropitious pathological sequence, the most prominent manifestation of diabetic peripheral neuropathy, leads to amputation of the diabetic foot. ${ }^{20-24}$ The refractory chronic ulcers are commonly associated with mobile and fixed rocker bottom foot. There is plenty of literature on the reconstruction of the rocker bottom foot using internal and external hardwares, inert bone grafts, and various flaps. ${ }^{9}$ Also, there are many reported series on the use of the pedicled fibula flap in posttraumatic peri-ankle defects. ${ }^{25-29}$ Similarly, the author speculated that the use of a vascularized fibula flap would facilitate stable foot reconstruction in unstable infected diabetic rocker bottom foot. The author's use of distally based pedicled fibula flap for reconstruction and salvage of the infected unstable rocker bottom diabetic foot in a staged manner is the first of its kind. The author had devised a protocol to salvage these types of veritable neuropathy consummated diabetic foot, using distally based pedicled fibula flap. This article analyzes the outcomes of the author's protocol.

\section{Aim}

The aim of this article is to evaluate the functional outcomes of the author's protocol of distally based pedicled fibula flap in staged reconstruction of Broadsky type 1 mobile and fixed rocker bottom diabetic Charcot foot with osteomyelitic destruction of the distal row of tarsal bones.

\section{Patients and Methods}

This retrospective study was conducted from 2002 to 2016. Twenty-eight patients (19 males and 8 females) with the intertarsal joint degeneration and tarsometatarsal joint degeneration (with sequestration of the distal row of tarsal bones with rocker bottom deformity) who had undergone staged reconstruction with distally based pedicled fibula flaps were included in the study exercising the following selection and exclusion criteria. Institutional ethical committee approval was obtained for the study. Informed consent was obtained from all patients (for the display of photographs and use of the clinical material in research).

\section{Selection Criteria}

1. Patients with Broadsky ${ }^{19}$ type1 CNAP and in Eichenholtz ${ }^{23}$ acute development or coalescence stage diabetic foot.

2. Those with osteomyelitic destruction of the tarsometatarsal region with pedal instability and refractory to medical therapy (for osteomyelitis and CNAP).

3. Those with was a plantar ulcer or sinus with a probe to bone or joint test positive (Wagner ${ }^{24}$ grade 3 )

\section{Exclusion Criteria}

1. Peripheral vascular disease and ischemic component.

2. Severe secondary infection leading to compromise of the overlying soft tissues and tarsal skin.
3. When other modalities of reconstruction used for stabilizing and reconstructing the arches without pedicled fibula flap.

4. Dialysis dependent patients and with severe Monckeberg's sclerosis.

5. Mentally unstable and uncooperative patients.

6. Patients who had lost follow-up in the postoperative period.

7. Patients with other types of Charcot's or in the combination with type 1 .

\section{Surgical Protocol}

\section{Preoperative Preparation}

All patients had a thorough clinical examination by the members of the multispecialty integrated team (plastic surgeon, orthopaedic surgeon, anesthetist, podiatrist, orthotist, rehabilitation nurse, and social worker) at the diabetic foot care clinic and the complete diagnosis arrived. All the key members of the team also simultaneously examined digital X-rays, pedobarograms, and magnetic resonance imaging (MRI) of the foot and ankle. They were treated with antibiotics (directive antibiotics based on the antibiogram obtained from bone culture study), bisphosphonates, calcium, and vitamin D supplements for 4 weeks before surgery. They were all trained to walk with walkers globally offloading the affected foot to prevent further progression of the disease in the insensate foot. After optimizing locoregional and general conditions, they were taken up for surgery.

\section{Stage 1}

Under axial regional anesthesia, with tourniquet control, sterile draping of leg and foot was done. Attention was paid to all faulty biomechanics. Clean surgeries were performed first. If there were faulty recruitment of peroneal musculatures, the tendon switch of peroneus brevis to peroneus longus was performed. Percutaneous tendoachilles tendon lengthening was done to rectify the triceps surae musculotendinous unit shortening contracture. Tibialis anterior muscle recruitment was also common and it was addressed by tenotomy. In diabetic neuropathy, muscle imbalances are common and were deduced by clinical examination and pedobarogram preoperatively. They were the precipitating and perpetuating factors for CNAP. Unless these were performed as an integral part of reconstruction recurrence was the rule. Then through medial and lateral incisions along tarsum, all sequestrated osseous elements and suppurated tissues were excised. Most of the time this resulted in complete or partial excision of the distal row of tarsal bones and the involved capsules and ligaments in the immediate vicinity. Samples of the osseous tissue were again sent for microbiological examination. After thorough wash and inspection of the cavity for any residual sepsis, the intraoperative pathology was carefully studied using fluoroscopy. The foot was stabilized with prebuilt circular external fixators (our modification of TAYLORS ${ }^{30}$ spatial frame) and subsequently, smooth wires were inserted. The external construct contained a one-foot ring/plate connected to the leg construct. The leg construct had two rings, one was proximal or distal to mid tibial diaphysis and another one was at distal tibial metaphysis with minimum three threaded connecting rods. The footplate/ 
ring was attached to the calcaneal tuberosity and the metatarsal necks using two crossing smooth wires at each point of fixation. Then the stirrup wire was inserted through the neck of the talus and attached to the adjacent tibial ring. The avenues for distraction at the distal tarsal level and arthroereisis were created using posts, washers, and plates extending from the footplate/ring. The intraoperative final check was done with fluoroscopy. In all these cases in this series (secondarily infected type 1 cases of Charcot's), there were fractures and subluxations invariably accompanied the infective sequestration of distal row of the tarsal bones. So, after the debridement there was actually little or no void because of the collapse and overriding of the osseous elements. During the stage 2, this was created progressively by distraction and arthroereisis maneuvers, by the modified Taylors frame. So in stage 1, all sequestrations and sepsis were debrided and foot was stabilized on the external ring fixator.

\section{Stage 2}

The distraction and arthroereisis were performed progressively. Weekly X-rays and postoperative wound care were provided. Patients were ambulated on the walker, globally offloading the operated foot from the second postoperative day. The strut activation and arthroereisis protocol were individualized and performed until the desired configurations of the foot and Meary angle reduction were achieved. At the final point, the void was created for the insertion of the vascularized fibula flap. All these were achieved in an average of 12 weeks. The deformities were reduced and an osseous void was obtained in stage 2 .

\section{Stage 3}

After sound healing of the medial and lateral tarsal wounds, the distally based pedicled fibular flap was harvested ( - Video 1) and inserted into the void created by distraction in the distal tarsal region and fixed with multiple axial Kirschner wires or cannulated screws along the axis of the metatarsal bone. Usually by the time of surgical intervention the void, which was created by the distraction and arthroereisis maneuvers, was filled with combination of granulation and fibrous tissues. Later were reamed out before the insertion of the vascularized fibula flap. A uniplanar bidirectional external fixator was also used to support the foot and leg in a plantigrade manner for 12 weeks. As these pedal bones were more osteoporotic and poor implant bearing quality author practice beaming of arches with long segment stabilization, quite contrast to Association for the Study of Internal Stabilization (ASIF) principle of short segment stabilization. The author had used inert corticocancellous bone graft from the harvested filleted distal fibula if needed, but not routinely. In stage 3, long segment stabilization was achieved with the incorporation of the vascularized fibula in the arches of the foot.

\section{Video 1}

Intraoperative video showing distally based fibula flap. Online content including video sequences viewable at: https://www.thieme-connect.com/products/ejournals/ html/10.1055/s-0040-1719197.

\section{Post Procedure Protocol}

Upon removal of the external fixators and Kirschner wires, the patient was trained to bear weight partially for another 2 months with customized removable Charcot restrained orthotic walker that was rendered nonremovable. Digital X-ray examination was done at 2 monthly intervals. The radiological bony consolidation was recorded for each patient separately. With proper gait training, they were allowed to weight bear on their legs after clinical and radiological consolidation. Patients were continued with bisphosphonates, vitamin D, and calcium supplements for 6 months postoperatively. They were instructed to walk with locoregional offloading silicone gel socks and contact surface area increasing footwears. This treatment algorithm and protocol were depicted in - Fig. 1

\section{Assessment}

At the end of the follow-up period, the functional outcome of the patient was assessed with the Musculoskeletal Tumor Society Rating (MSTSR) score system by two independent observers (one podiatrist and another one was orthopaedic surgeon not involved in this research). Each one independently assessed the X-rays, Pedobarograms, and the gait, and the score was finally assigned. At the end of the average follow-up period, the final computed MSTSR score was arrived and analyzed.

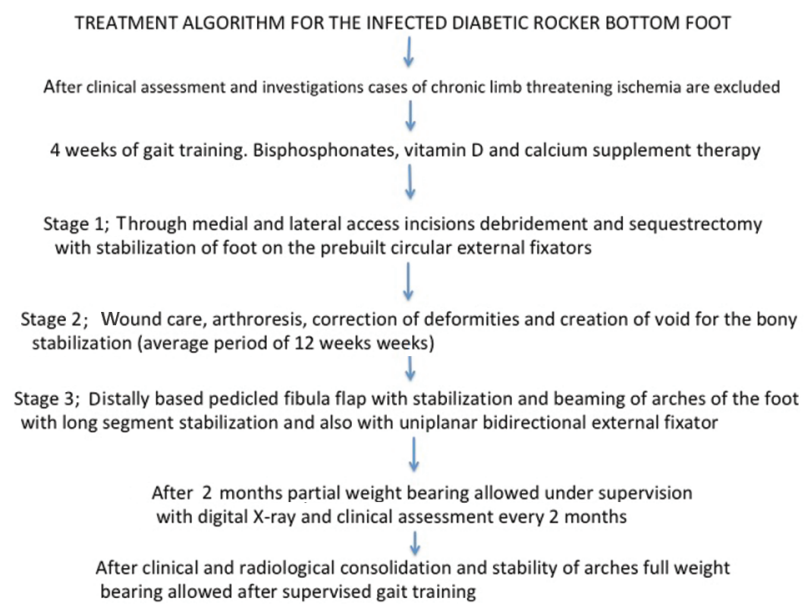

Fig. 1 Treatment algorithm for the staged reconstruction of infected rocker bottom diabetic foot. 


\section{Case 1 Illustration of Mobile Infected Diabetic Rocker Bottom Foot}

Thirty-nine years old male patient, a known case of type 2 diabetes mellitus, presented to the diabetic foot care clinic with left mobile rocker bottom foot (-Fig. 2). There were sinuses on the medial and lateral aspect of tarsum, pus and bony spicules discharge for 3 months (-Fig. 2). He had noticed sudden swelling of his left foot following an alcohol binge 8 months ago. He could not recall any event of injury. It was managed conservatively with leg elevation and graded compression bandage in a private clinic. The swelling subsided after a month. He never had pain or any constitutional symptoms at that time. Five months later, he had recurrent swelling of the left foot followed by blisters on either side of the tarsum leading to sinus formation. Clinical examination revealed no other systemic manifestations of diabetes mellitus except bilateral severe peripheral neuropathy and loss of protective sensation. Focused pedal examination revealed mobile rocker bottom foot on weight-bearing with the collapse of medial cuneiform-navicular joint and faulty biomechanics of pronation of foot with peroneal recruitment (-Figs. 2 and 3 ). There was crepitus on the passive movement of the tarsal joint with increased mobility at the level of the tarsometatarsal joint. The foot was unstable on weight-bearing. The anteroposterior dimension of the foot was also found to be shortened (-Fig. 2). Pedal pulses were well felt on both feet. On probing the sinuses, it led to the mobile osseous sequestered elements. On milking along with the sinuses, there was a discharge of sanguineous white thick pus with a greenish tinge. Digital X-rays revealed complete sequestration of the distal row of tarsal bones (-Fig. 3) and edematous surrounding tissue planes. His laboratory data revealed anemia, elevated erythrocyte sedimentation rate, raised liver enzymes, and prothrombin time with prolonged international normalized ratio. Pedobarogram revealed a wide hyperpression area on the sole of the left foot with collapsing arches with the center of gravity falling away from the tarsum on standing and walking (-Fig. 3). Directive antibiotic therapy based on the antibiogram was started. His general condition was medically optimized with a multispecialty integrated approach. He was counseled for drinking habits. Because of CNAP he was started on bisphosphonate therapy 3 months before the surgery and was continued during the staged surgical treatment. He had undergone staged reconstruction as mentioned earlier ( for 30 months. He had pain free gait with regained stable arches of the left foot at the end of follow-up (-Fig. 6). The follow-up pedobarogram ( - Fig. 7) showed stable arch formation and the follow-up digital X-rays revealed consolidated arched of the foot with a well-healed fibula (-Fig. 6). At the end of the follow-up, he was assessed by the MSTSR score (-Fig. 8).

\section{Case 2 Illustration of Fixed Infected Diabetic Rocker Bottom Foot}

A 50-year-old male presented with Broadsky type 1 Eichenholtz coalescent stage CNAP with pus discharging sinus anteromedial to the ankle joint (-Figs. 9 and 10). He had bounding pedal pulses. He had 5 months of preoperative bisphosphonate therapy. His digital X-ray (-Fig. 10) confirmed the clinical diagnosis. MRI revealed a large loculation at the naviculocuneiform junction. (-Fig. 11). After preoperative preparation for 1 month, the patient had undergone staged (stages 1-3) salvage reconstruction that lasted 3 months duration (-Fig. 11). He had radiological bony consolidation at 7.5 months postoperatively. Ultimately it resulted in the shoe-able plantigrade stable foot at 27 months postoperatively (-Fig. 11).
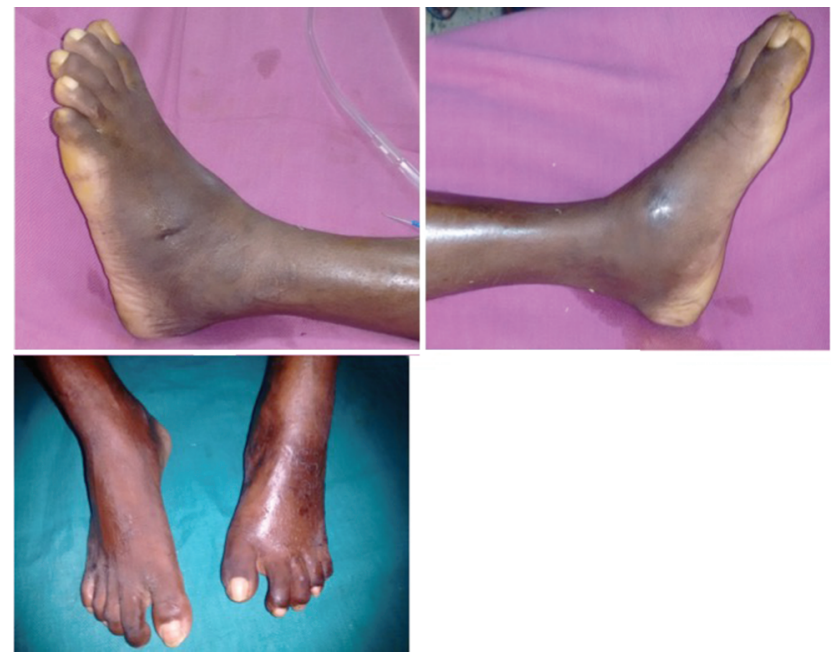

Fig. 2 Case 1: (upper two) preoperative image showing left mobile rocker bottom foot. (lower center) Preoperative image shows the shortening of left foot segment when compared with right.

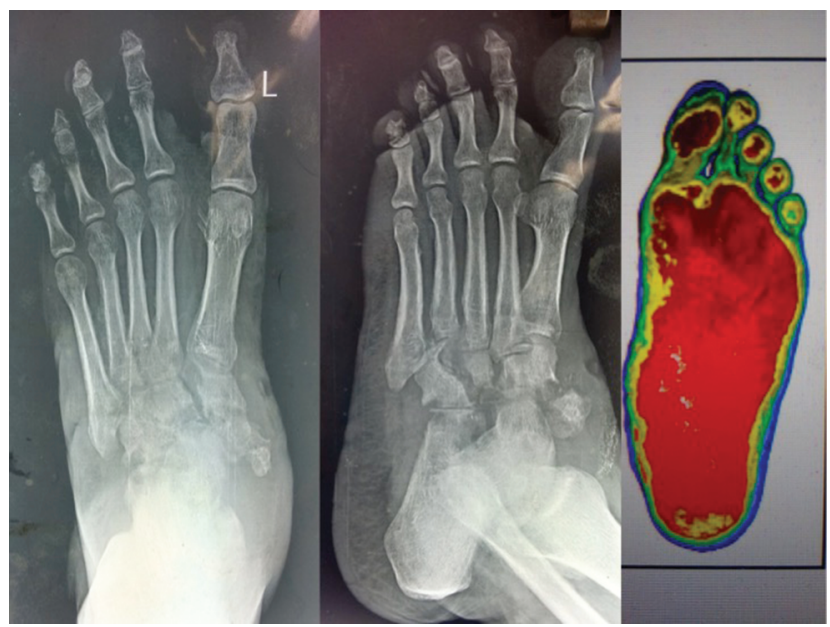

Fig. 3 Case 1: (left two) digital X-rays of left foot showing complete destruction of distal row of tarsal bones. (right extreme) The preoperative pedobarography showing the collapsing arches and hyperpression involving the whole sole. 


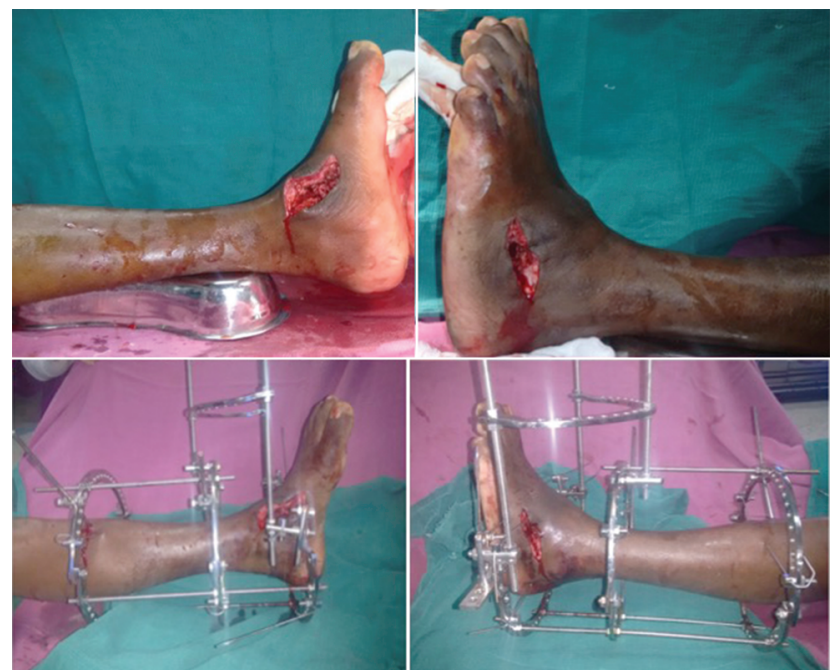

Fig. 4 Case 1: (upper two) intraoperative images after sequestrectomy and debridement through medial and lateral access incisions. (lower two) Foot is being stabilized with ring external fixator containing stirrups and struts.
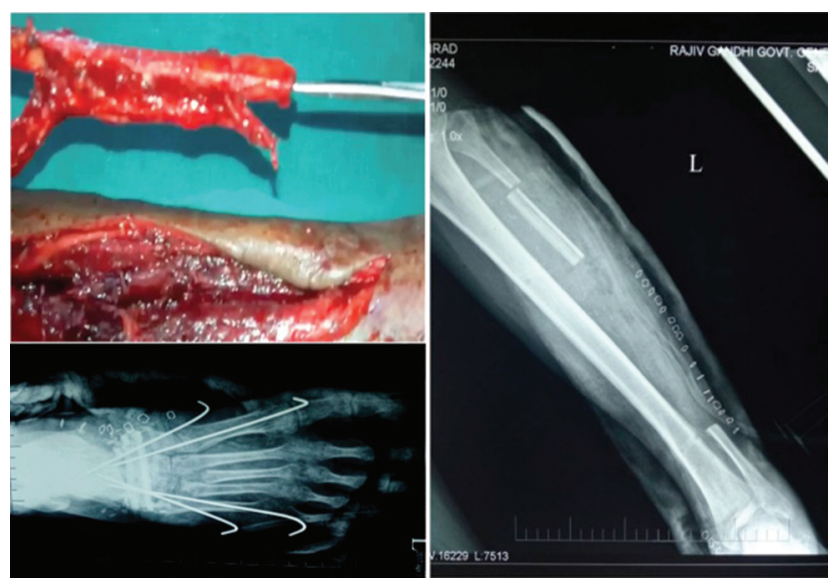

Fig. 5 Case 1: image showing (left upper) pedicled fibula flap. (right upper) Postoperative digital X-ray showing fibula donor site defect. (left below) Long segment fixation of pedicled fibula in the tarsal void.

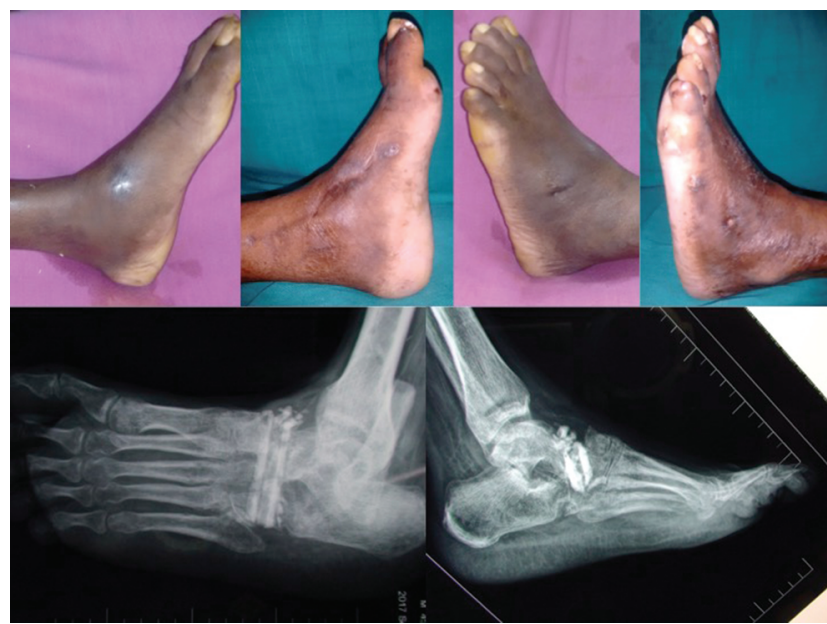

Fig. 6 Case 1: (upper row) 2-year postoperative image showing well-retained arches with pre- and postoperative views for comparison. (lower row) Two-year postoperative digital X-rays showing consolidated fibula maintaining the beaming of arches and Meary line.
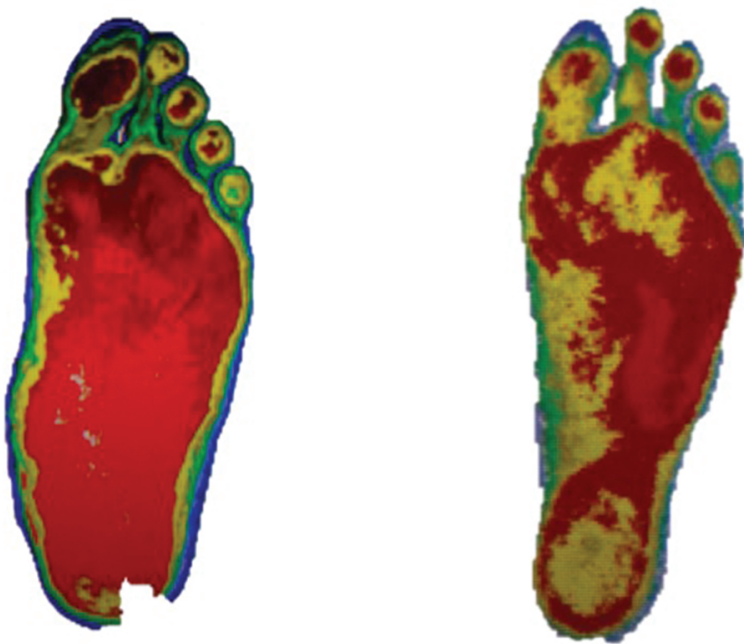

Fig. 7 Case 1: preoperative and late postoperative pedobarogram compared.

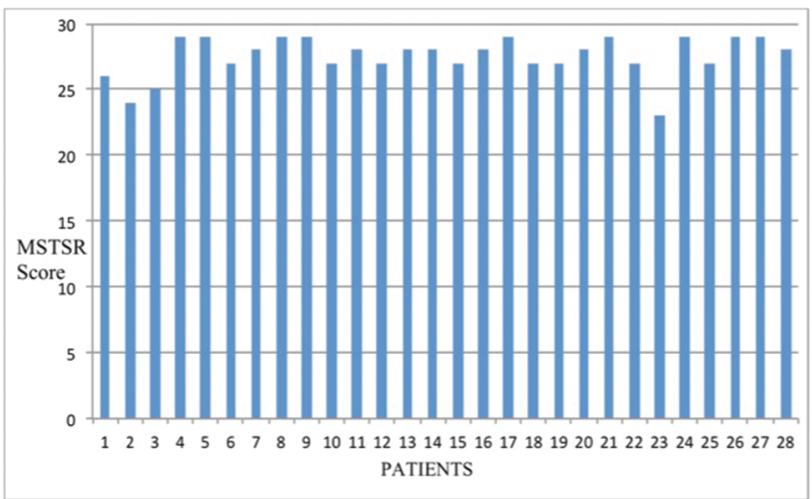

Fig. 8 Musculoskeletal Tumor Society Rating (MSTSR) scoring of patients who had undergone staged reconstruction of infected diabetic rocker bottom foot with distally based pedicled fibula flap.

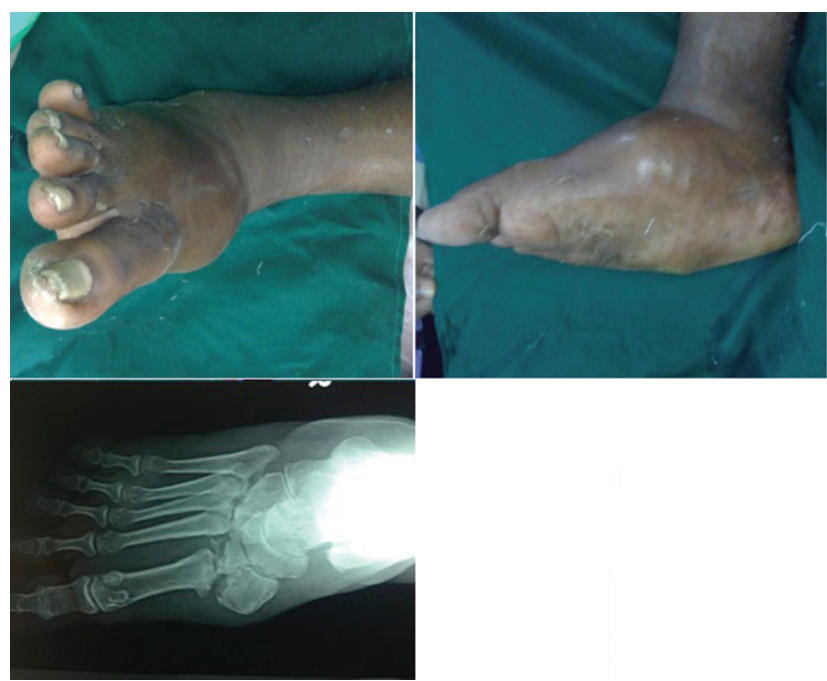

Fig. 9 Case 2: (upper row) right side infected fixed rocker bottom foot. (lower) Digital X-ray revealing the osteomyelitic destruction of distal row of tarsal bones. 


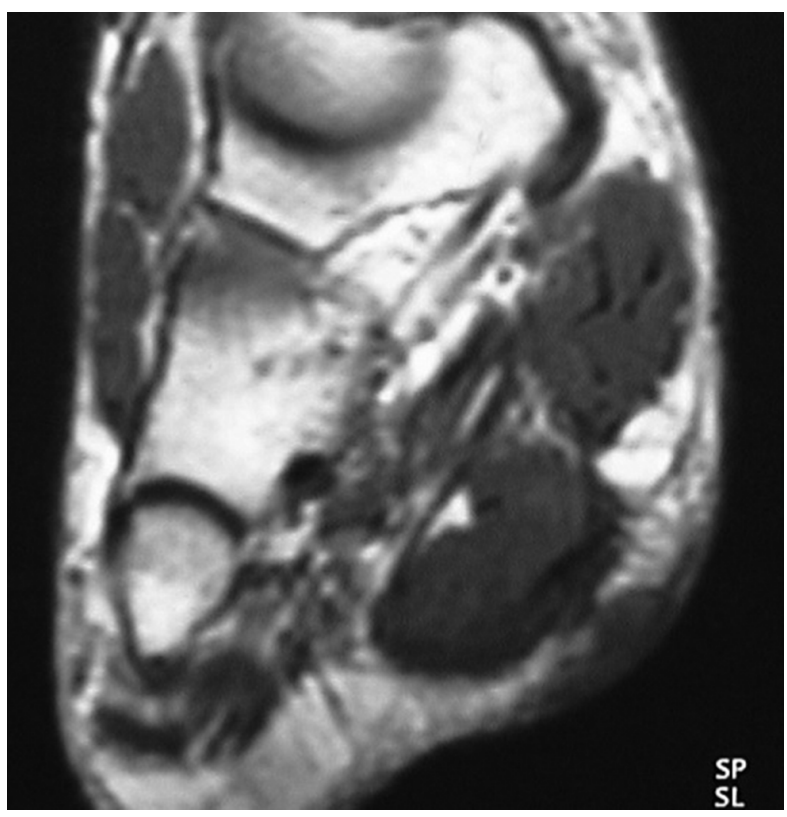

Fig. 10 Case 2: Magnetic resonance imaging T2-weighted images revealing the subluxed and destroyed medial cuneiform and navicular bones.
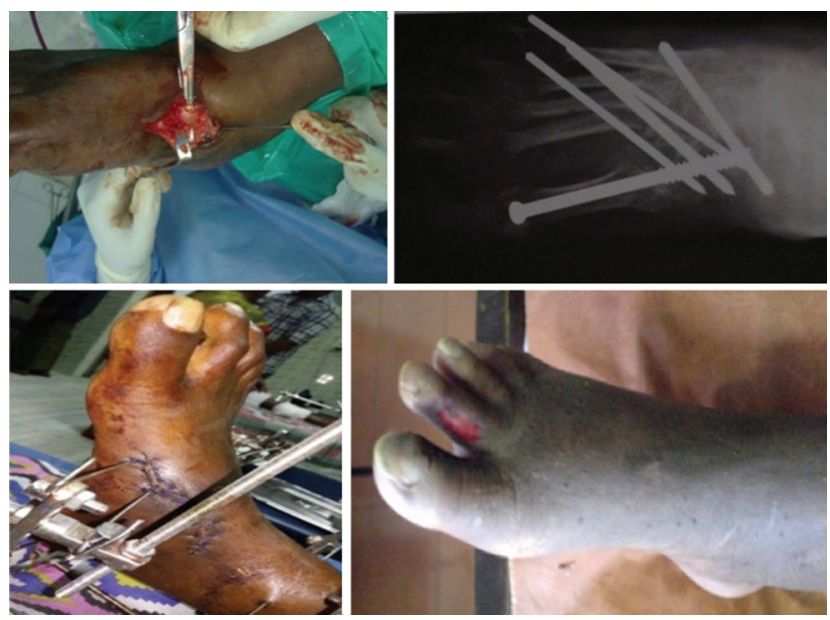

Fig. 11 Case 2: (upper row left) intraoperative picture showing access to debridement. (upper row right) Stage 3 digital X-ray showing long segment stabilization with $4 \mathrm{~cm}$ long distally based fibula graft after stage 1 and stage 2 corrections. (lower row left) Stabilization with external fixator in stage 3 (lower right) 26 months late postoperative picture with well-maintained arches (erosion at first web due to ill-fitting footwear).

\section{Results}

Clinical data and results of 28 patients are given in - Table 1 . The average age of the patients was 45.5 years. There were 20 males and 8 females. All the patients had unilateral involvement (left side 21 and right side 7). Conservative treatment was given for an average period of 3.5 months. As adjunct procedures, percutaneous tendoachilles lengthening with Peroneus longus to Peroneus brevis tendon switch were done in 20 patients $(71.4 \%)$ and percutaneous tendoachilles lengthening alone was done in 8 patients (28.5\%).
The average size of the pedicled fibula harvested was 6.67 $\mathrm{cm}$. In the treatment, average duration from stage 1 to 3 completion was 3.5 months. The average time taken for the consolidation of reconstructed arches with radiological evidence was 6.4 months. Patients were followed up for an average period of 30.5 months. Complications occurred in three patients (10.7\%). One patient had pin site infection and another patient had a recurrent infection of the fibula, and both were treated conservatively with antibiotics. Both patients recovered well. One patient had a fracture and recurrence of Charcot's disease (3.5\%). He was managed with medical therapy and conservative measures. On assessment with the MSTSR score at the end of the follow-up period, the

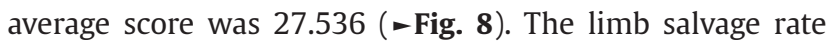
with the author's procedure was $96.4 \%(p=0.045)$. Author series has nil recurrence of plantar ulcers or deformities at Broadsky type 1 during the follow-up period.

\section{Discussion}

CNAP has multifactorial etiopathogenesis. The diabolic interactions between the various components of diabetic peripheral neuropathy are as follows: (1) Sensory neuropathy results in loss of protective sensation and joint sensation, (2) motor neuropathy predisposes to deformity due to muscle imbalance, and (3) autonomic neuropathy precipitates hyperemic bony demineralization and redundancy of ligaments.

It is a challenging and time-consuming job to reconstruct the secondarily infected, CNAP devastated, diabetic rocker bottom feet. The orthoplastic reconstructive science is well advanced that it has settled the controversy ${ }^{31,32}$ whether patients can be taken up for the reconstruction, in the acute developmental or coalescence stage. Now the staged reconstruction is the standard of care for these patients who are unable to stand or walk because of the deformity with instability and poor general health due to deep-seated sepsis. Recidivism and transfer lesions can occur in up to $15 \%$ of these patients ${ }^{33}$ treated by modalities that do not incorporate proper internal offloading techniques to ameliorate all the faulty biomechanics. The author's protocol had integrated two novel innuendos, namely (1) the integral treatment of all faulty biomechanics otherwise called internal offloading or surgical offloading, (2) beaming of arches with long segment stabilization.

1. The integral internal offloading techniques in addition to the skeletal reconstruction and stability restoration were done in all cases (-Table 1). "Diagnose before treating" is the golden commandment of Ralph Millard Jr. ${ }^{34}$ Failure to recognize all the faulty biomechanics especially in a mobile deformity of the diabetic foot is the common cause of recurrence of lesions. Therefore, correct diagnosis, which delineates all the faulty biomechanics, and correct treatment plan directed toward each faulty biomechanics are an integral part of the author's internal or surgical offloading technique. This is responsible for the high limb salvage rate of $96 \%$ in this series. Also, it resulted in the nil recurrence of plantar ulcers in the author's series. Ingredients of the protocol were individualized for each 
Table 1 Clinical data of patients who had undergone staged reconstruction of infected diabetic rocker bottom foot with distally based pedicled fibula flap

\begin{tabular}{|c|c|c|c|c|c|c|c|c|c|}
\hline $\begin{array}{l}\text { SI. } \\
\text { No }\end{array}$ & Age & Sex & $\begin{array}{l}\text { Duration of conser- } \\
\text { vative and medical } \\
\text { treatment }(\mathrm{mo})\end{array}$ & $\begin{array}{l}\text { Size of the } \\
\text { pedicled } \\
\text { fibula }(\mathrm{cm})\end{array}$ & $\begin{array}{l}\text { Adjunct } \\
\text { procedures }\end{array}$ & $\begin{array}{l}\text { Time taken for } \\
\text { consolidation of } \\
\text { reconstructed } \\
\text { arches with } \\
\text { radiological } \\
\text { evidence (mo) }\end{array}$ & $\begin{array}{l}\text { Follow-up } \\
\text { period (mo) }\end{array}$ & Complications & $\begin{array}{l}\text { MSTSR } \\
\text { score }\end{array}$ \\
\hline 1 & 40 & M & 3.5 & 6 & PTL+PL-PB TS & 6.5 & 21 & - & 26 \\
\hline 2 & 50 & $\mathrm{M}$ & 5 & 8 & PTL only & 7 & 37.5 & - & 24 \\
\hline 3 & 52 & $M$ & 2 & 7.5 & PTL+PL-PB TS & 6 & 32 & - & 25 \\
\hline 4 & 49 & $\mathrm{M}$ & 4.5 & 6.5 & PTL only & 5 & 25 & - & 29 \\
\hline 5 & 46 & $\mathrm{~F}$ & 3 & 6 & PTL+PL-PB TS & 6.5 & 43 & - & 29 \\
\hline 6 & 42 & $\mathrm{M}$ & 4 & 6.5 & PTL+PL-PB TS & 5 & 34 & - & 27 \\
\hline 7 & 43 & $\mathrm{~F}$ & 2.5 & 6.5 & PTL only & 7.5 & 19.5 & - & 28 \\
\hline 8 & 47 & $\mathrm{~F}$ & 5 & 6 & PTL+PL-PB TS & 6 & 46 & $\begin{array}{l}\text { External pin tract } \\
\text { infection treated } \\
\text { with antibiotics }\end{array}$ & 29 \\
\hline 9 & 51 & $\mathrm{M}$ & 3 & 7.5 & PTL+PL-PB TS & 5.5 & 36 & - & 29 \\
\hline 10 & 38 & $\mathrm{M}$ & 2 & 6.5 & PTL+PL-PB TS & 7 & 30 & - & 27 \\
\hline 11 & 42 & $\mathrm{M}$ & 2.5 & 6 & PTL+PL-PB TS & 6 & 39.5 & - & 28 \\
\hline 12 & 48 & $\mathrm{~F}$ & 5.5 & 6.5 & PTL only & 5 & 17 & - & 27 \\
\hline 13 & 51 & $\mathrm{M}$ & 3 & 8 & PTL+PL-PB TS & 7 & 26 & - & 28 \\
\hline 14 & 39 & M & 2 & 6 & PTL+PL-PB TS & 6 & 37 & $\begin{array}{l}\text { Recurrent } \\
\text { infection of fibula, } \\
\text { treated with anti- } \\
\text { biotics and settled } \\
\text { conservatively }\end{array}$ & 28 \\
\hline 15 & 46 & M & 3 & 6 & PTL+PL-PB TS & 5 & 29 & - & 27 \\
\hline 16 & 50 & M & 5 & 4 & PTL+PL-PB TS & 7.5 & 27 & - & 28 \\
\hline 17 & 48 & $\mathrm{~F}$ & 3.5 & 6.5 & PTL only & 8 & 18.5 & - & 29 \\
\hline 18 & 41 & $\mathrm{M}$ & 2 & 8 & PTL+PL-PB TS & 5 & 41 & - & 27 \\
\hline 19 & 39 & $\mathrm{~F}$ & 3 & 6 & PTL+PL-PB TS & 7 & 30 & - & 27 \\
\hline 20 & 47 & M & 5 & 6.5 & PTL+PL-PB TS & 5.5 & 19.5 & - & 28 \\
\hline 21 & 43 & $\mathrm{M}$ & 4.5 & 7.5 & PTL only & 8 & 30 & - & 29 \\
\hline 22 & 49 & $\mathrm{M}$ & 3.5 & 8 & PTL+PL-PB TS & 7 & 39 & - & 27 \\
\hline 23 & 58 & $\mathrm{~F}$ & 2 & 7 & PTL only & 6.5 & 16 & $\begin{array}{l}\text { Fracture and } \\
\text { recurrence of } \\
\text { Charcot disease }\end{array}$ & 23 \\
\hline 24 & 45 & M & 4 & 6.5 & PTL+PL-PB TS & 8 & 28.5 & - & 29 \\
\hline 25 & 40 & $M$ & 2 & 7 & PTL+PL-PB TS & 5 & 35 & - & 27 \\
\hline 26 & 44 & $\mathrm{M}$ & 4 & 6.5 & PTL only & 7.5 & 35 & - & 29 \\
\hline 27 & 41 & $\mathrm{~F}$ & 4 & 7 & PTL+PL-PB TS & 6 & 29 & - & 29 \\
\hline 28 & 45 & M & 5 & 6.5 & PTL+PL-PB TS & 8 & 33 & - & 28 \\
\hline
\end{tabular}

Abbreviations: F, female; M, male; MSTSR, Musculoskeletal Tumor Society Rating score; PTL, percutaneous tendoachilles lengthening; PL-PB TS, peroneus longus to peroneus brevis tendon switch.

patient depending upon the pathology and faulty biomechanics. The author's current treatment protocol evolves from the setbacks and recidivism failures following isolated exostectomy and local flap cover techniques. ${ }^{35,36}$

2. Beaming of arches with long segment stabilization was done in all cases. Reconstructive surgery of the diabetic CNAP rocker bottom foot typically entails stabilization and/ or arthroereisis of multiple collapsed joints. Complications like hardware construct failure, recurrent infections, recurrence of ulcers, and deformities are reported in the reconstruction of Charcot's from 15 to $20 \%$ in various series. ${ }^{19,37}$ The use of a well-vascularized osseous element-vascularized pedicled fibula flap for the beaming of the arches of the foot-brings down the complication rate to $10.7 \%$ in the author's series. As these pedal bones were more osteoporotic and poor implant bearing quality author practice beaming of arches with long segment stabilization, quite contrast to the ASIF principle of short segment stabilization. The transversely placed vascularized fibula flaps with anti-infective and large remodeling potential reinforce 
both the longitudinal and transverse arches of the foot on healing. Thus, the vascularized fibula flap not only added the bony stock but also facilitated beaming of the arches

Lower extremity amputation is known to affect a significant increase in cardiovascular output, which is highly relevant in the patient population affected by CNAP. ${ }^{9}$ Therefore, saving the foot and limb imposes less metabolic stress on coronary atherosclerosis restrained heart. So, the author's subjective contention is "saving the foot is saving the life of the patient." 38

There is no doubt that the free fibular flap represents excellent options for single-stage reconstruction of complex defects in foot. ${ }^{39}$ Compared with free fibular flap transfer, the pedicled fibula transfer has following distinct advantages in the reconstruction of rocker bottom diabetic foot. (1) It is microvascular surgery minus microvascular anastomosis and therefore freedom from complications of circulatory compromises. ${ }^{28}(2)$ In the ipsilateral limb, anterior and posterior tibial vessels are completely spared and therefore the rare dreadful complication of amputation of a foot due to arterial thrombosis extending on to the main vessel ${ }^{28}$ is not a complication in the author series. (3) Peroneal vessels are disease-free many times in diabetic. ${ }^{40,41}$ (4) Shorter learning curve. (5) Technically easier with less arduous flap monitoring postoperatively. (6) It does not envisage quality vessels in the inflammatory zone for its survival. (7) Last but not the least, the pedicled fibular flap is harvested in the ipsilateral leg, which prevents extra morbidity on the healthy side. But nevertheless, the long pedicle of the fibula flap with reverse venous flow envisages no pressure or kinking or twisting in the tunnel. The author's subjective feeling is "planning in reverse" helps a long way in eschewing this complication. The author's distally based pedicled fibula flap is based on the large anastomotic communication branch between the peroneal vessels and the posterior tibial vessels as the peroneal vessels run very close to the interosseous membrane $\sim 2.5 \mathrm{~cm}$ proximal to the tibiofibular syndesmosis. ${ }^{42}$ The author's forethoughtful multidisciplinary evaluation in each case is responsible for the relatively good to excellent outcome in this series for the following reasons: (1) The risk-benefit ratio of lower extremity reconstruction and salvage versus amputation is fundamental and is analyzed before each case selection. (2) The anatomically conceived procedures address the faulty biomechanics in all cases as an integral part of reconstruction. (3) Customized external ring fixators with avenues for correction of all the skeletal and soft-tissue abnormalities are used in each case. (4) Well, vascularized osseous element-the distally pedicled fibula flap is used for the beaming and reconstruction of foot. This culls infection and provides a superior ossification environment for the early stable outcome. These three stages are needed in the reconstruction of infected diabetic rocker bottom foot. Stage 1 and 2 need to eschew infection completely and to create skeletal void with correction of deformities and stage 3 with the attainment of the local tissue homeostasis beaming of arches with distally based pedicled fibula flap was done.

To this date, there is no study on the staged reconstruction for salvaging the infected rocker bottom diabetic foot in the literature to the best of our knowledge. The author's study is first of its kind in the clinical scenario. The limitation of the author's study is of small scale and neuroischemic diabetic foot cases are excluded. The strength of the study is good-to-excellent outcome shown in the staged reconstruction using pedicled fibula flap for beaming and stabilizing the infected Charcot diabetic foot. It is well known that all these reconstructive procedures are palliative and the disease can again set in the other regions of the diabetic foot. Even in the author's series, there was one case recurrence of Charcot's arthropathy in the subtalar joint in the follow-up. So, the authors staged algorithm in the salvaging the infected diabetic rocker bottom foot fetched good results in the follow-up period. It needs a long-term study to draw more useful conclusions.

\section{Conclusion}

Contemporary astute surgeons must be aware of the fact that the population of diabetic Charcot's foot is burgeoning with an increase in the prevalence of diabetic neuropathy. In carefully selected patients with infected rocker bottom diabetic foot, this protocol of staged reconstruction using distally based pedicled fibula flap may fetch good results with fewer complications. It is well known that all these reconstructive procedures are palliative and the disease can again set in the other region of the diabetic foot. The author's protocol for staged reconstruction and salvage of the neuropathy ravaged infected diabetic rocker bottom foot using the pedicled fibula flap will be a new addendum in the reconstructive armamentarium of orthoplastic approach. It needs a long-term and large-size study to draw more useful conclusions.

\section{Declaration of Patient Informed Consent}

The authors certify that they have obtained all appropriate patient consent forms. In the form, the patient(s) has/have given his/her/their consent for his/her/their images and other clinical information to be reported in the journal. The patients understand that their names and initials will not be published and due efforts will be made to conceal their identity.

\section{Ethical Approval}

The institutional ethical committee has approved the clinical study

\section{Financial Support and Sponsorship \\ Nil.}

\section{Conflicts of Interest}

All the coauthors including the corresponding author declare that they have no conflicts of interest.

\section{References}

1 Charcot JM. Sur quelques arthropathies qui paraissent dependre d'une lesion du cerveau ou de la moelle epiniere. Arch Physiol Norm Pathol 1868;1:161-178

2 Narres M, Kvitkina T, Claessen H, et al. Incidence of lower extremity amputations in the diabetic compared with the non-diabetic population: a systematic review. PLoS One 2017;12(8):e0182081

3 International Diabetes Federation, IDF Diabetes Atlas. 7th edition. Belgium: International Diabetes Federation; 2016 
4 Trautner C, Haastert B, Spraul M, Giani G, Berger M. Unchanged incidence of lower-limb amputations in a German City, 1990-1998. Diabetes Care 2001;24(5):855-859

5 Almaraz MC, González-Romero S, Bravo M, et al. Incidence of lower limb amputations in individuals with and without diabetes mellitus in Andalusia (Spain) from 1998 to 2006. Diabetes Res Clin Pract 2012;95(3):399-405

6 David G. Amstrong @\# Diabetes. Available from: http://www. bit. ly/2Knl6b3. Accessed March 2019

7 Laclé A, Valero-Juan LF. Diabetes-related lower-extremity 26 amputation incidence and risk factors: a prospective seven-year study in Costa Rica. Rev Panam Salud Publica 2012; 32(3):192-198

8 Ang Y, Yap CW, Saxena N, Lin LK, Heng BH. Diabetes-related lower extremity amputations in Singapore. Proc Singapore Healthc 2017;26:76-80

9 Capobianco CM, Ramanujam CL, Zgonis T. Charcot foot reconstruction with combined internal and external fixation: case report. J Orthop Surg Res 2010;5(1):7

10 de Souza LJ. Charcot arthropathy and immobilization in a weight-bearing total contact cast. J Bone Joint Surg Am 2008; 90(4):754-759

11 Pinzur MS. Current concepts review: Charcot arthropathy of the foot and ankle. Foot Ankle Int 2007;28(8):952-959

12 Wang JC, Le AW, Tsukuda RK. A new technique for Charcot's foot reconstruction. J Am Podiatr Med Assoc 2002;92(8):429-436

13 Balakrishnan TM, Pramasivam I, Thirunavukarasu K, Janardhanan J, Narayanan S. Limb salvage using microvascular reconstructions for secondary regional vascular insufficiency in the neuro ischemic diabetic foot; is it making impact? Indian J Vasc Endovasc Surg 2019;6:65-73

14 Salini D, Harish K, Minnie P, et al. Prevalence of Charcot arthropathy in Type 2 diabetes patients aged over 50 years with severe peripheral neuropathy: a retrospective study in a Tertiary Care South Indian Hospital. Indian J Endocrinol Metab 2018;22(1):107-111

15 Sohn MW, Lee TA, Stuck RM, Frykberg RG, BudimanMak E. Mortality risk of Charcot arthropathy compared with that of diabetic foot ulcer and diabetes alone. Diabetes Care 2009;32(5):816-821

16 Rogers LC, Frykberg RG, Armstrong DG, et al. The Charcot foot in diabetes. Diabetes Care 2011;34(9):2123-2129

17 Frykberg RG, Belczyk R. Epidemiology of the Charcot foot. Clin Podiatr Med Surg 2008;25(1):17-28, v

18 Rajbhandari SM, Jenkins RC, Davies C, Tesfaye S. Charcot neuroarthropathy in diabetes mellitus. Diabetologia 2002;45(8): 1085-1096

19 Trepman E, Nihal A, Pinzur MS. Current topics review: Charcot neuroarthropathyofthefootandankle.FootAnkleInt2005;26(1): 46-63

20 Tesfaye S, Vileikyte L, Rayman G, et al; Toronto Expert Panel on Diabetic Neuropathy. Painful diabetic peripheral neuropathy: consensus recommendations on diagnosis, assessment and management. Diabetes Metab Res Rev 2011;27(7):629-638

21 Callaghan BC, Cheng HT, Stables CL, Smith AL, Feldman EL. Diabetic neuropathy: clinical manifestations and current treatments. Lancet Neurol 2012;11(6):521-534

22 Quattrini C, Tesfaye S. Understanding the impact of painful diabetic neuropathy. Diabetes Metab Res Rev 2003; 19(Suppl 1):S2-S8
23 Eichenholtz S, Charcot Joints. Springfield, IL: Charles C. Thomas; 1966

24 Oyibo SO, Jude EB, Tarawneh I, Nguyen HC, Harkless LB, Boulton AJ. A comparison of two diabetic foot ulcer classification systems: the Wagner and the University of Texas wound classification systems. Diabetes Care 2001;24(1):84-88

25 Fekete A, Gáspár S, Szűcs A. New way to reconstruct severe injuries to the lower extremity. J Plast Surg Hand Surg 2014; 48(3):216-218

26 Chung YK, Chung S. Ipsilateral island fibula transfer for segmental tibial defects: antegrade and retrograde fashion. Plast Reconstr Surg 1998;101(2):375-382, discussion 383-384

27 Kwok AC, Tatro E, Jones KB, Agarwal JP. Pedicled retrograde fibula flap for ankle reconstruction after oncologic resection of the distal fibula. Plast Reconstr Surg Glob Open 2017;5(2):e1232

28 Wang CY, Han P, Chai Y-M, Lu S-D, Zhong W-R. Pedicled fibular flap for reconstruction of composite defects in foot. Injury 2015;46(2):405-410

29 Lykoudis EG, Dimitrios P, Alexandros BE. One-stage reconstruction of the complex midfoot defect with a multiple osteotomized free fibular osteocutaneous flap: case report and literature review. Microsurgery 2010;30(1):64-69

30 Keshet D, Eidelman M. Clinical utility of the Taylor spatial frame for limb deformities. Orthop Res Rev 2017;9:51-61

31 Myerson MS, Henderson MR, Saxby T, Short KW. Management of midfoot diabetic neuroarthropathy. Foot Ankle Int 1994;15(5):233-241

32 Papa J, Myerson M, Girard P. Salvage, with arthrodesis, in intractable diabetic neuropathic arthropathy of the foot and ankle. J Bone Joint Surg Am 1993;75(7):1056-1066

33 Armstrong DG, Todd WF, Lavery LA, Harkless LB, Bushman TR. The natural history of acute Charcot's arthropathy in a diabetic foot specialty clinic. Diabet Med 1997;14(5):357-363

34 Saraf S. Millard's 33 commandments of plastic surgery. The Internet J Plastic Surg 2006;4(1):x

35 Pinzur MS, Sage R, Stuck R, Kaminsky S, Zmuda A. A treatment algorithm for neuropathic (Charcot) midfoot deformity. Foot Ankle 1993;14(4):189-197

36 Zgonis T, Roukis TS, Lamm BM. Charcot foot and ankle reconstruction: current thinking and surgical approaches. Clin Podiatr Med Surg 2007;24(3):505-517, ix

37 Resch S. Corrective surgery in diabetic foot deformity. Diabetes Metab Res Rev 2004;20(Suppl 1) :S34-S36

38 Balakrishnan TM, Ilayakumar P. Microvascular reconstruction in the revascularised diabetic foot: a perforosome approach. Clin Res Foot Ankle 2016;4:206

39 Yazar S, Lin CH, Wei FC. One-stage reconstruction of composite bone and soft-tissue defects in traumatic lower extremities. Plast Reconstr Surg 2004;114(6):1457-1466

40 Dibble $\mathrm{JH}$, The Pathology of Limb Ischemia. Edinburgh: Oliver\&Boyd; 1966

41 Dosluoglu HH, Cherr GS, Lall P, Harris LM, Dryjski ML. Peroneal artery-only runoff following endovascular revascularizations is effective for limb salvage in patients with tissue loss. J Vasc Surg 2008;48(1):137-143

42 Attinger CE, Evans KK, Bulan E, Blume P, Cooper P. Angiosomes of the foot and ankle and clinical implications for limb salvage: reconstruction, incisions, and revascularization. Plast Reconstr Surg 2006;117(7, Suppl):261S-293S 